This is a postprint version of the following published document:

D'Auria, Bernardo and Kanta, Spyridoula (2015). Pure threshold strategies for a two-node tandem network under partial information. Operations Research Letters, v. 43, n. 5, pp. 467-470. Available in: http://dx.doi.org/10.1016/j.orl.2015.06.014

(c) Elsevier

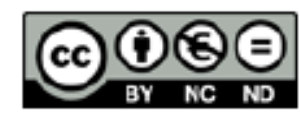

This work is licensed under a Creative Commons Attribution-NonCommercialNoDerivatives 4.0 International License. 


\title{
Pure threshold strategies for a two-node tandem network under partial information
}

\author{
Bernardo D'Auria ${ }^{\mathrm{a}, \mathrm{b}, *}$, Spyridoula Kanta ${ }^{\mathrm{a}}$ \\ a Statistics Department, Madrid University Carlos III, Leganés, Spain \\ ${ }^{\mathrm{b}}$ ICMAT, Madrid University Carlos III, Leganés, Spain
}

\section{ABSTRACT}

In a two node tandem network, customers decide to join or balk by maximizing a given profit function whose costs are proportional to the sojourn time they spend at each queue. Assuming that their choices are taken without knowing the complete state of the system, we show that a pure threshold equilibrium policy exists. In particular we analyze the case when the partial information consists in informing the arrival customers of the total number of users in the network.

\section{Keywords:}

Tandem network

Individual

optimization Partial

information

Threshold strategy

\section{Introduction}

Queueing literature is recently devoting an increasing attention to the economic analysis of queueing systems. Indeed in real applications it is not uncommon that the input to a queueing system is not exogenously defined and is the result of the combined effect of the decisions made by the arriving customers. They may decide whether to join or balk the system according to their convenience and these choices in general lead to a final equilibrium. This phenomenon is mathematically modeled by assuming they are rationally optimizing a given individual profit function. This research, started in the 70 s by $[8,4]$, now has reached a good maturity, two central monographs are $[6,11]$. Most of the literature focuses on a single server system, while we focus here on network models, in particular a series of two $\mathrm{M} / \mathrm{M} / \cdot$ queues. Previous studies have looked at parallel queues $[12,5,7]$ and for more general topologies extensive studies have been done in the field of telecommunications, see [3,9]. A close model is [1], where a series of queues of $\mathrm{M} / \mathrm{M} / \mathrm{m}$ types is analyzed and the form of the symmetric customer equilibrium is derived together with the explicit socially optimal strategies. The main difference with our model is that there customers make their decisions without getting any information on the state of the system, while here they know

\footnotetext{
Corresponding author at: Statistics Department, Madrid University Carlos III, Leganés, Spain.

E-mail address: bdauria@est-econ.uc3m.es (B. D’Auria).
}

the total number of customers already inside. Usually network models show an intrinsic difficulty in getting explicit results, and this partially explains a relatively scarcer literature. The two node tandem network that we study has the advantage of being simpler and allowing a complete analysis. Customers make the decisions to balk or join after knowing how many customers are already in the network. In real applications, it is common that people do not know the complete information on the state of the system, as usually this information is shortly summarized to simplify the decision process. Examples may be found in healthcare systems, where treatment requires two different steps, such as a first queue to get a doctor reservation and a second queue to be attended by the doctor. The interesting result is that the partial information setting simplifies drastically the analysis, allowing to get for this specific case explicit results.

The model is introduced in Section 2, we compute in Section 3 the expected sojourn time of an arriving customer assuming that the full state of the system is known. In Section 4 the same analysis is done when the arriving customers are informed about the total number of customers in the network. Finally we compute the equilibriumstrategyin Section 5 .

\section{The model}

We consider a tandem network with two single server nodes with infinite buffers and service times independent and exponentially distributed. Using the index $I$, with $I=1$ or 2 , to refer to the first or the second node, we denote by $\mu_{l}$ the service 
rate at node $l$. Customers arrive to the system according to a Poisson process with rate $\lambda$ and before joining the network they receive partial information about the state of the system. The state space is $\mathbb{N}^{2}$, that is all possible pairs $\left(Q_{1}, Q_{2}\right)$ with $Q_{l}$ the queue length at node $l$. A tagged customer that just arrives, gets a reword $R$ for joining the network, and pays for each unit of sojourn time at node $l$ a cost $C_{l}$ with a resulting random profit given by $P=R-C_{1} S_{1}-$ $C_{2} S_{2}$, where $S_{l}$ denotes the sojourn times she would spend at node $l$.

The tagged user makes her decision by optimizing the expected profit given the information she receives at her arrival time, $k=$ $Q_{1}+Q_{2}$, that is

$P_{K}(k)=R-C_{1} T_{K, 1}(k)-C_{2} T_{K, 2}(k)$,

with $T_{K, l}=\mathbb{E}_{K}\left[S_{l} \mid Q_{1}+Q_{2}=k\right]$. The subindex $K$ tells that the rest of the population is using a pure threshold strategy with threshold $K$ in joining the queue. That is all users besides the tagged one join the network if and only if it contains less than $K$ customers.

The main result of the paper is to show that the tandem network admits a pure threshold $K$, that is there exists a $K \in \mathbb{N}$ such that

$P_{K}(k) \geq 0$ as $k<K$ and $P_{K}(k)<0$ as $k \geq K$.

Remark 1. By using the subindex $K$, we are implicitly assuming that the rest of the population is not allowed to use strategies different from a pure threshold one. This assumption is not restrictive for our purposes, but it does not preclude the existence of policies (even of equilibrium type) that are of a different form.

Remark 2. We always assume that $R>C_{1} / \mu_{1}+C_{2} / \mu_{2}$. Being this relation false, a user would get negative net profit even joining an empty network implying a unique equilibrium given by the empty system.

Before analyzing the described model, we first study the case when the complete information is available to the arriving customers. This is done in the next section.

\section{Mean sojourn times}

Let $S_{l}(n, m)$ be the sojourn time spent at queue $l$ by a tagged customer that joins a system being in state $(n-1, m)$, that is she is going to occupy position $n$ in the first queue. Let $T_{l}(n, m)=$ $E\left[S_{l}(n, m)\right]$ be the corresponding expectation and $T(n, m)=$ $T_{1}(n, m)+T_{2}(n, m)$ the total expected sojourn time. The sojourn time in the first queue is Erlang distributed, that is $S_{1}(n, m) \sim$ $\operatorname{Erlang}\left(n, \mu_{1}\right)$ with mean $T_{1}(n, m)=n / \mu_{1}$. The total sojourn time can be computed recursively by applying a first step analysis, that leads to the following formula,

$$
\begin{aligned}
T(n, m)= & \frac{1}{\mu_{1}+\mu_{2}}+\frac{\mu_{1}}{\mu_{1}+\mu_{2}} T(n-1, m+1) \\
& +\frac{\mu_{2}}{\mu_{1}+\mu_{2}} T(n, m-1), \quad n, m>0 .
\end{aligned}
$$

The second term on the right hand side of (2) considers a potential departure from the first queue and the last term a potential departure from the second queue. These events occur with probability $\mu_{l} /\left(\mu_{1}+\mu_{2}\right), l=1,2$ respectively. To complete the recursion the following boundary conditions are needed

$T(0, m)=\frac{m}{\mu_{2}} ; \quad T(n+1,0)=\frac{1}{\mu_{1}}+T(n, 1), \quad n, m>0$.

Using (2) we get a recursive formula to compute $T_{2}(n, m)$ as shown in the following lemma.
Lemma 3. The expected sojourn time at the second queue, $T_{2}(n, m)$, can be computed with the following recursive formula

$$
\begin{aligned}
& T_{2}(n, m)=\left(\frac{\mu_{2}}{\mu_{1}+\mu_{2}}\right)^{m} T_{2}(n-1,1) \\
& \quad+\frac{\mu_{1}}{\mu_{1}+\mu_{2}} \sum_{k=0}^{m-1}\left(\frac{\mu_{2}}{\mu_{1}+\mu_{2}}\right)^{k} T_{2}(n-1, m+1-k)
\end{aligned}
$$

valid for $n>0$ and $T_{2}(0, m)=m / \mu_{2}$, with $m \geq 0$.

Proof. By (2), we get that $T_{2}(n, m)$ satisfies the following recursive equation

$$
\begin{aligned}
T_{2}(n, m)= & \frac{\mu_{1}}{\mu_{1}+\mu_{2}} T_{2}(n-1, m+1) \\
& +\frac{\mu_{2}}{\mu_{1}+\mu_{2}} T_{2}(n, m-1), \quad n, m>0,
\end{aligned}
$$

and by (3), similar boundary conditions are satisfied. By induction argument it is then straightforward to verify that (4) holds true.

The following lemma characterizes the conditions under which the $T$-functions are monotone non-decreasing in the variable $n$. These conditions are important for the analysis of Section 5.

Lemma 4. The functions $T_{1}(n, m)$ and $T(n, k-n)$ are non decreasing in $n$. The function $T_{2}(n, m)$ is non decreasing in $n$ if and only if $\mu_{1} \geq$ $\mu_{2}$.

Proof. The statement is obvious for $T_{1}(n, m)$ that does not depend on $m$.

One way to show that the function $T(n, k-n)$ is non decreasing in $n$, for $n \leq k$ is by proving that $T(n+1, m) \geq T(n, m+$ 1) by induction using Eqs. (2)-(3). We prefer to use a coupling argument. Using the same probability space, we construct two networks starting respectively with $(n+1, m)$ and $(n, m+1)$ initial users. The proof follows by comparing the waiting times of the customers that are the last ones in the first queue of both networks, and showing that the one in the former network waits more than the corresponding one in the latter. To construct the coupling we assume that the service times for all customers are the same in both networks but we move the customer in service at the first queue of the first network at the end of the queue of the second node of the second network. Since the exit times are ordered by the FIFO discipline and because the moved customer reduces its sojourn time by her service time in the first node, the result holds.

Finally to show that $T_{2}(n, m)$ is non decreasing in $n$ we prove that $\Delta_{1} T_{2}(0, m) \geq 0$ for all $m$ where $\Delta_{1} T_{2}(n, m)=T_{2}(n+1, m)-$ $T_{2}(n, m)$. From $(4)$, the following holds for any $n>0$ and $m \geq 0$,

$$
\begin{aligned}
\Delta_{1} T_{2}(n, m)= & \left(\frac{\mu_{2}}{\mu_{1}+\mu_{2}}\right)^{m} \Delta_{1} T_{2}(n-1,1)+\frac{\mu_{1}}{\mu_{1}+\mu_{2}} \\
& \times \sum_{k=0}^{m-1}\left(\frac{\mu_{2}}{\mu_{1}+\mu_{2}}\right)^{k} \Delta_{1} T_{2}(n-1, m+1-k) .
\end{aligned}
$$

If $\Delta_{1} T_{2}(0, m) \geq 0$ the same holds for $n>0$ as all the coefficients in (5) are positive. In the opposite case $T_{2}(n, m)$ is clearly decreasing for some value of $(n, m)$. Let $\alpha=\mu_{1} / \mu_{2}$, one can check that

$\Delta_{1} T_{2}(0, m)=\frac{1}{\mu_{2}}\left(\frac{\alpha-1+(\alpha+1)^{-m}}{\alpha}\right)$.

The quantity above is decreasing in $m$. To check that it would be non negative for any value of $m$ we take $m \rightarrow \infty$ and get the required condition $\alpha \geq 1$. 


\section{Expected sojourn times under the $K$-strategy}

We assume that all arriving customers receive the partial information about the state of the network and decide to join only if the number of customers in the system, say $k$, is less than a given threshold $K \geq 0$. Under the $K$-strategy the tandem network behaves as a semiopen Jackson network, see [2, Section 2.3]. Let

$Q_{l}^{*}$ be the stationary random number of customers at node $l$ and $Q^{*}=Q_{1}{ }^{*}+Q_{2}{ }^{*}$ be the stationary total number of customers in the queue. The stationary distribution is given by, see [2, Theorem 2.5],

$\pi_{K}(n, m)=\mathbb{P}_{K}\left(Q_{1}^{*}=n, Q_{2}^{*}=m\right)=c_{K} \rho_{1}^{n} \rho_{2}^{m}, \quad n+m \leq K$

where $\rho_{l}=\lambda / \mu_{l}$ and $c_{K}^{-1}=\sum_{n+m \leq K} \rho_{1}^{n} \rho_{2}^{m}$ is the normalization constant.

Assuming $n \leq K$, the conditional probability $\mathbb{P}_{K}\left(Q^{*}=n \mid Q^{*}=\right.$ $k)=\rho_{l}^{n} \rho_{3-l}^{k-n} / \sum_{h=0}^{k} \rho_{l}^{h} \rho_{3-l}^{k-h}$ does not depend on $K$. Let $p_{l}(n \mid k)=$ $\mathbb{P}_{K}\left(Q_{l}^{*}=n \mid Q^{*}=k\right)$, after algebraic manipulation, we get

$p_{l}(n \mid k)= \begin{cases}\mu_{l}^{k-n} \mu_{3-l}^{n}\left(\mu_{1}-\mu_{2}\right) /\left(\mu_{1}^{k+1}-\mu_{2}^{k+1}\right) & \mu_{1} \neq \mu_{2} \\ 1 /(1+k) & \mu_{1}=\mu_{2} .\end{cases}$

The independence from $K$ allows to consider the random variables $Q_{l}^{*}(k), l \in\{1,2\}$, having distributions $p_{l}(\cdot \mid k)$ and not depending on the pure threshold strategy employed by all customers.

Remark 5. The assumption that the rest of the population follows a threshold policy is necessary in order to have the steady state distribution expressed in the form given in (7).

Let us define by $T_{l}(k)=\mathbb{E}\left[S_{l} \mid Q^{*}=k\right]$ the expected sojourn time at queue $l$ of a tagged customer that enters a system containing $k$ customers.

Lemma 6. Assuming $\mu_{1} \neq \mu_{2}$, it holds that

$T_{1}(k)=\frac{1}{\mu_{1}-\mu_{2}}-\frac{k+1}{\mu_{1}} \frac{\mu_{2}^{k+1}}{\mu_{1}^{k+1}-\mu_{2}^{k+1}}$

$T_{2}(k)=\left(1-\frac{\mu_{2}}{\mu_{1}}\right) \frac{\mu_{1}^{k+1}}{\mu_{1}^{k+1}-\mu_{2}^{k+1}} \sum_{n=0}^{k} T_{2}(n+1, k-n)\left(\frac{\mu_{2}}{\mu_{1}}\right)^{n}$

and for $\mu_{1}=\mu_{2}, T_{1}(k)=1 / \mu_{1}(1+k / 2)$ and $T_{2}(k)=1 /(k+$ 1) $\sum_{n=0}^{k} T_{2}(n+1, k-n)$.

Proof. By definition $T_{1}(k)=1 / \mu_{1} \quad \sum_{n=0}^{k}(n+1) p_{1}(n \mid k)$, therefore (7) with $\mu_{1} \neq \mu_{2}$ implies

$$
\begin{aligned}
T_{1}(k) & =\frac{1}{\mu_{1}} \frac{\mu_{1}-\mu_{2}}{\mu_{1}^{k+1}-\mu_{2}^{k+1}} \sum_{n=0}^{k}(n+1) \mu_{1}^{k-n} \mu_{2}^{n} \\
& =\frac{1}{\mu_{1}} \frac{\mu_{1}^{k}\left(\mu_{1}-\mu_{2}\right)}{\mu_{1}^{k+1}-\mu_{2}^{k+1}} \frac{\mu_{1}^{2+k}-(2+k) \mu_{1} \mu_{2}^{1+k}+(k+1) \mu_{2}^{2+k}}{\mu_{1}^{k}\left(\mu_{1}-\mu_{2}\right)^{2}} .
\end{aligned}
$$

Simplifying the expression above we get (8). The formula for $T_{2}(k)$ is obtained similarly by the expression $T_{2}(k)=\sum_{n=0}^{k} T_{2}(n+1, k-$ n) $p_{1}(n \mid k)$. The results for $\mu_{1}=\mu_{2}$ can be obtained in a similar way or more directly by noticing that in this case the random variables $Q_{1}^{*}$ are discrete uniformly distributed on $\{0,1, \ldots, k\}$. One could also compute the limit of the expressions (8) and (9) as $\mu_{1} \rightarrow \mu_{2}$.

\section{Threshold equilibrium strategy}

By Lemma 6 the expected profit of a tagged customer receiving the information $k$, given in (10), does not depend on the strategy
$K$ employed by the rest of customers. We can therefore compute it as

$P(k)=R-C_{1} T_{1}(k)-C_{2} T_{2}(k)$.

The tagged customer decides to enter only if $P(k) \geq 0$. In the sequel we show under what conditions the expected net profit function is decreasing in $k$, moreover since this function is constant with respect to the strategy $K$, we obtain that the equilibrium strategy is in addition a dominant strategy in the class of threshold strategies. Before stating the main result we require the following lemma on the stochastic monotonicity of the random variables $Q_{i}^{*}(k)$.

Lemma 7. The random variables $Q_{l}^{*}(k)$ are increasing stochastically ordered in $k \geq 0$.

Proof. In order to show that $Q_{1}^{*}(k+1) \geq_{s t} Q_{1}^{*}(k)$ it is enough to prove the stronger condition given by the likelihood ratio ordering, see [2]. This last condition can be checked by proving that

$$
\begin{aligned}
& \mathbb{P}\left\{Q_{l}^{*}(k+1)=n+1\right\} \mathbb{P}\left\{Q_{l}^{*}(k)=n\right\} \\
& \quad \geq \mathbb{P}\left\{Q_{l}^{*}(k+1)=n\right\} \mathbb{P}\left\{Q_{l}^{*}(k)=n+1\right\}
\end{aligned}
$$

for any $n \geq 0$. It is easy to check that (11) holds as equality for any $n<k$ and is a strict inequality for $n=k$ where the second term is 0 and therefore the result holds true.

Remark 8. We explicitly note that the result of Lemma 7 is different from requiring that the variables $Q^{*}$ are stochastically ordered with respect to the strategy $K$. This result refers to non conditional quantities and could be proved by a coupling argument similar to the one used in [10].

Lemma 7 implies the following monotonicity result to the mean sojourn functions.

Lemma 9. The functions $T_{1}(k)$ and $T(k)$ are non decreasing for all values of the ratio $\mu_{1} / \mu_{2}$. The function $T_{2}(k)$ is non decreasing when this ratio is greater than 1 .

Proof. The function $T_{1}(k)$ is non decreasing by Lemma 7 and because $T_{1}(n, m)$ is non decreasing in $n$ as well. For $T(k)$, it follows by the following

$$
\begin{aligned}
T(k+1) & =\mathbb{E}\left[T\left(Q_{1}^{*}(k+1), k+1-Q_{1}^{*}(k+1)\right)\right] \\
& \geq \mathbb{E}\left[T\left(Q_{1}^{*}(k+1), k-Q_{1}^{*}(k+1)\right)\right] \\
& \geq \mathbb{E}\left[T\left(Q_{1}^{*}(k), k-Q_{1}^{*}(k)\right)\right]=T(k)
\end{aligned}
$$

where in the last equality we used the fact that $T(n, k-n)$ is non decreasing, see Lemma 4 , and the stochastic monotonicity of the variables $Q_{1}{ }^{*}(k)$, proved in Lemma 7. A similar argument works for the function $T_{2}(k)$. Assuming $\mu_{1} \geq \mu_{2}$, we have

$$
\begin{aligned}
T_{2}(k+1) & =\mathbb{E}\left[T_{2}\left(k+1-Q_{2}^{*}(k+1), Q_{2}^{*}(k+1)\right)\right] \\
& \geq \mathbb{E}\left[T_{2}\left(k-Q_{2}^{*}(k+1), Q_{2}^{*}(k+1)\right)\right] \\
& \geq \mathbb{E}\left[T_{2}\left(k-Q_{2}^{*}(k), Q_{2}^{*}(k)\right)\right]=T_{2}(k) .
\end{aligned}
$$

The first inequality follows by Lemma 4 under the assumption on the service rates, the second inequality follows by the monotonicity of $Q_{2}{ }^{*}(k)$, shown in Lemma 7, and the fact that $T_{2}(k-$ $m, m)$ is non decreasing in $m$ for any fixed $k>0$.

To see that $T_{2}(k)$ may decrease when $\mu_{1}<\mu_{2}$, let us consider $\mu_{1}=0.1$ and $\mu_{2}=1$, then

\begin{tabular}{llllll} 
& $k=1$ & $k=2$ & $k=3$ & $k=4$ & $k=5$ \\
\hline$T_{2}(k)$ & 0.198347 & 0.0313319 & 0.00438963 & 0.000573903 & 0.0000717892.
\end{tabular}

Corollary 10. If $\mu_{1}>\mu_{2}$ or if $C_{1} \geq C_{2}$ the expected net profit, $P(k)$, is non increasing. 
Proof. The result follows from Lemma 9 if $\mu_{1}>\mu_{2}$. If $C_{1} \geq C_{2}$, it is enough to rewrite the profit function as $P(k)=R-\left(C_{1}-C_{2}\right) T_{1}(k)-$ $C_{2} T(k)$, and use the monotonicity of $T_{1}(k)$ and $T(k)$.

Finally we state the main result that gives the conditions to find the strategy that induces the Nash equilibrium.

Theorem 11. When the information known by the arriving customers is the total number of customers in the network only, then the equilibrium strategy is given by the threshold $K$ such that

$K=\arg \min \{k \in \mathbb{N}: P(k)<0\}$.

According to this strategy a tagged customer enters only if she finds less than $\mathrm{K}$ customers in the system. The K-strategy is a dominant strategy in the class of threshold strategies.

Proof. Let the index $K$ be the one obtained by formula (14), including $K=\infty$. We show that the strategy $K$ is the best response against itself. The system is always ergodic, therefore with no loss of generality we assume it starts empty. Since all customers employ the $K$-strategy the tagged user will never find more than $K$ customers in the system and according to (14), she will follow the same strategy, and the result follows.

The actions that the tagged customer may take for the values of $k>K$ are irrelevant as she will never find the system in these states. However if the monotonicity conditions given in Lemma 9 hold, the $K$-strategy leads to a subgame perfect equilibrium, see [6].

The $K$-strategy is dominant because it is the best response to any other possible threshold strategy. This holds because the net profit function does not depend neither on the arrival rate nor on the threshold employed by other customers. Assuming that the system is working under a pure strategy with a threshold different from $K$, if at some point in time customers start to behave selfishly, they will all adopt the $K$-strategy. If the monotonicity conditions of Lemma 9 are not satisfied, this statement uses the fact that the Markov chain is ergodic and it hits almost surely the null state.

The expression (10) depends on the values of the function $T_{2}(n, m)$ given in (9) and as such we cannot expect to obtain a closed formula for the equilibrium threshold strategy. However we can always compute it numerically by (9).

\section{Conclusions}

This work analyzes the equilibrium behavior of a tandem network when customers may choose the actions of balking or joining the system by taking into account economic considerations. This paper is the first to look at queues in series, and the surprising result is that if users only receive partial information on the status of the network, in particular the total number of customers in the system, a pure strategy exists. It may be the case that such result may be extended to more general network topologies, this will be the subject of future research.

\section{Acknowledgments}

The research of the first author is partially supported by the Spanish Ministry of Education and Science Grants MTM201342104-P (FEDER funds), MTM2010-16519 and RYC-2009-04671. The authors would like to thank the anonymous referee for the insightful comments that helped in improving the correctness of the statement of the result.

\section{References}

[1] A. Burnetas, Customer equilibrium and optimal strategies in Markovian queues in series, Ann. Oper. Res. 208 (1) (2013) 515-529.

[2] H. Chen, D.D. Yao, Fundamentals of Queueing Networks, Springer-Verlag, 2001.

[3] C. Courcoubetis, R. Weber, Pricing Communication Networks: Economics, Technology and Modelling, in: Wiley Interscience Series in Systems and Optimization, John Wiley \& Sons, 2003.

[4] N.M. Edelson, K. Hildebrand, Congestion tolls for Poisson queueing processes, Econometrica 43 (1975) 81-92.

[5] R. Hassin, On the advantage of being the first server, Manage. Sci. 42 (4) (1996) 618-623.

[6] R. Hassin, M. Haviv, To Queue or Not to Queue: Equilibrium Behavior in Queueing Systems, Kluwer, Boston, 2003.

[7] M. Haviv, R. Zlotnikov, Computational schemes for two exponential servers where the first has a finite buffer, RAIRO-Oper. Res. 45 (1) (2011) 17-36.

[8] P. Naor, The regulation of queue size by levying tolls, Econometrica 37 (1969) $15-24$.

[9] T. Roughgarden, Selfish Routing and the Price of Anarchy, The MIT Press, 2005

[10] Y. Sakuma, M. Miyazawa, On the effect of finite buffer truncation in a two-node Jackson network, J. Appl. Probab. 42 (1) (2005) 199-222. 03.

[11] S. Stidham, Optimal Design of Queueing Systems, CRC Press, Boca Raton, 2009.

[12] W. Whitt, Deciding which queue to join: Some counterexamples, Oper. Res. 34 (1) (1986) 55-62. 